\title{
Incorporation of dietary fibre-rich oyster mushroom (Pleurotus sajor-caju) powder improves postprandial glycaemic response by interfering with starch granule structure and starch digestibility of biscuit
}

\author{
Sze Han Ng ${ }^{\text {a }}$, Sathyasurya Daniel Robert ${ }^{b}$, Wan Amir Nizam Wan Ahmad $\quad$ c, Wan Rosli Wan Ishak ${ }^{\mathrm{a}, \Uparrow}$ \\ ${ }^{a}$ Nutrition Program, School of Health Sciences, Universiti Sains Malaysia Health Campus, 16150 Kubang Kerian, Kelantan, Malaysia \\ ${ }^{\mathrm{b}}$ Dietetic Program, School of Health Sciences, Universiti Sains Malaysia Health Campus, 16150 Kubang Kerian, Kelantan, Malaysia \\ ${ }^{\mathrm{c}}$ Biomedicine Program, School of Health Sciences, Universiti Sains Malaysia Health Campus, 16150 Kubang Kerian, Kelantan, Malaysia
}

\section{a bstract}

The purpose of this study was to determine the effects of Pleurotus sajor-caju (PSC) powder addition at 0 , 4,8 and $12 \%$ levels on the nutritional values, pasting properties, thermal characteristics, microstructure, in vitro starch digestibility, in vivo glycaemic index $(\mathrm{Gl})$ and sensorial properties of biscuits. Elevated incorporation levels of PSC powder increased the dietary fibre (DF) content and reduced the pasting viscosities and starch gelatinisation enthalpy value of biscuits. The addition of DF-rich PSC powder also interfered with the integrity of the starch granules by reducing the sizes and inducing the uneven spherical shapes of the starch granules, which, in turn, resulted in reduced starch susceptibility to digestive enzymes. The restriction starch hydrolysis rate markedly reduced the $\mathrm{Gl}$ of biscuits. The incorporation of $8 \%$ PSC powder in biscuits $(\mathrm{GI}=49)$ could be an effective way of developing a nutritious and low-GI biscuit without jeopardizing its desirable sensorial properties.

\section{Introduction}

The worldwide prevalence of diabetes is forecasted to increase from the current 382 million people to 592 million by 2035, indicating that approximately $10 \%$ of the population will have diabetes (Guariguata et al., 2014). This debilitating chronic metabolic disorder can be reversed through diet modification. Consumer demand for diabetes-related functional foods has increased enormously with the goal of improving their blood glucose control. A promising approach of examining the physiological effects of food is by the concept of glycaemic index $(\mathrm{Gl})$ which is a value assigned to foods with respect to their effect on post-meal glycaemia (Jenkins et al., 2008). As a result of the slow carbohydrate digestion and absorption rate, low-GI food produces a steady rise in blood glucose level and thus regarded as a meaningful dietary approach in the management of diabetes.

One of the major food components that can be utilised to reduce $\mathrm{GI}$ is dietary fibre (DF).

It has recently been gaining increasing

\footnotetext{
$\Uparrow$ Corresponding author.

E-mail addresses: juliensh1@hotmail.com(S.H. Ng), rajdorai511@yahoo.com (S.D. Robert), wanamir@usm.my (W.A.N. Wan Ahmad), wrosli@usm.my (W.R. Wan Ishak).
}

importance in view of its close relationship with the reduction of postprandial blood glucose response (Schuchardt et al., 2016). Apart from that, b-glucan, a soluble DF featuring the structural polysaccharides of D-glucose monomers, provides interesting health-promoting effects such as the reduction of blood glucose and blood cholesterol levels (Chen \& Raymond, 2008). Products used to enhance the DF and b-glucan content of foods traditionally come from cereals such as wheat, oat and barley. Recently, edible fungi have been gradually utilised as DF sources and innovated in the world markets due to their better nutritional qualities (Cheung, 2013).

Pleurotus sajor-caju (PSC) is an excellent consumable grey oyster mushroom characterised by a white spore print, gills attachment, and often with an eccentric stip or no stip at all (Cheung, 2013). It grows generally on stumps and trunks of deciduous trees, and nowadays it is also cultivated on decayed organic materials. Due to its ease of cultivation, annual production of Pleurotus spp. is more than 900,000 tons ( $14.2 \%$ of the total world mushroom production) and is ranked the second-most popular cultivated mushroom after the button mushroom (Imran, Raja, Basith, \& Asarudin, 2011). Interestingly, due to its pleasant taste and desirable pharmacological properties, it is useful for the culinary and medicinal purposes such as combating tumours, strengthening the immune 
system against diseases, lowering blood pressure, reducing blood glucose and decreasing blood cholesterol levels ( $\mathrm{Ng}$, Mohd Zain, Zakaria, Wan Ishak, \& Wan Ahmad, 2015; Shah et al., 2007).

Currently, research has been conducted on the application of PSC powder as a source of DF in wheat-based products (Wan Rosli \& Aishah, 2012). However, the potential role of PSC powder in the development of low-GI food has not yet been addressed in the scientific literature. Therefore, discovering the possible use of PSC powder as a novel low-GI food ingredient is of great interest and importance in functional food research. Biscuit is one of the largest starchy processed foods which mainly constituted of wheat flour, sucrose and fat. Due to its position on top 10 lists of daily consumed food (Norimah et al., 2008), it could be an effective carrier of DF by replacing wheat flour with PSC powder. Being a starchy food, the GI of biscuit highly depends on the starch gelatinisation, integrity of starch granules and its accessibility to starch-splitting enzyme. The present study was conducted to ascertain the effects of different incorporation levels of PSC powder on the key characteristics of biscuits including the nutritional values, pasting properties, thermal characteristics, microstructure, in vitro starch digestibility and in vivo $\mathrm{Gl}$ also carried out to determine the most Sensory evaluation was palatable PSC-enriched biscuit.

\section{Materials and methods}

There were control sample and three experimental samples. The control sample was the biscuit without addition of PSC powder whereas the experimental samples were the biscuits formulated with 4,8 and $12 \%$ of PSC powder to partially replace wheat flour. This study was conducted to compare the nutritional values, pasting properties, thermal characteristics, microstructure, in vitro starch digestibility, GI in vivo and sensorial properties of control biscuit and biscuit formulated with different levels of PSC powder.

\subsection{Sample preparation (PSC powder)}

Fresh fruiting bodies of PSC $(5 \mathrm{~kg})$ were purchased from the wet market located in Malacca Town, Malaysia and dried using the low-heat air-dried technique $\left(50-55^{\circ} \mathrm{C}\right)$ established by Anjaad Industries (M) Sdn. Bhd which is also located in Malacca Town, Malaysia. The dehydrated PSC (yield 10\% w/w) were milled into powder form by using an electrical blender (Waring Commercial 8010S, USA) and sifted into fine powder by using a sieve with diameter of $125 \mathrm{I} \mathrm{m}$. Then, the collected PSC powder was kept in screw cap bottles and stored at $4{ }^{\circ} \mathrm{C}$ until further use.

\subsection{Biscuit development}

Biscuits were developed by utilising various commercially available raw ingredients: butter $(140 \mathrm{~g})$, sugar $(86.4 \mathrm{~g})$, egg white $(20 \mathrm{~g})$, wheat flour $(270 \mathrm{~g})$, corn flour $(50 \mathrm{~g})$ and baking powder $(2 \mathrm{~g})$. PSC powder was utilised to partially replace wheat flour at $0,4,8$ and $12 \%$. In a mixing bowl, butter, sucrose and egg white were mixed together and beaten by using an electric hand mixer (National Inc., Osaka, Japan) until a creamy texture was achieved. After other dry ingredients were added to them, they were beaten again for $5 \mathrm{~min}$ to obtain the desired dough. The dough was sheeted on a flat surface to roughly $5 \mathrm{~mm}$ in thickness and formed into a round shape that was $3 \mathrm{~cm}$ in diameter. Next, the dough was put on a lightly greased baking sheet before being baked in an oven (Zanussi ZCG841 W, England) at $180^{\circ} \mathrm{C}$ for $14 \mathrm{~min}$. After that, the biscuits were cooled at room temperature for $1 \mathrm{~h}$ before being tested.

\subsection{Nutritional analyses}

2.3.1. Proximate composition, sucrose and calorific value analyses Proximate composition analyses were done following methods in accordance with the AOAC (1996) for moisture, total ash, fat, protein and carbohydrate. Based on AOAC 982.14 (AOAC, 1995), sucrose content was determined by applying high-pressure liquid chromatography (HPLC) with evaporative light scattering detection (ELSD). The calorific values $(\mathrm{kcal} / 100 \mathrm{~g})$ of the samples were determined by using a bomb calorimetry (IKA O C2000 Basic).

\subsubsection{DF compositions and b-glucan analyses}

The insoluble DF (IDF) and soluble DF (SDF) contents of the PSC powder and biscuit samples were determined by employing an enzymatic gravimetric method, based on the AOAC 991.42, AOAC 993.19 and AACC 32-21 (AOAC, 1990). For the total DF (TDF) content, it was calculated as a sum of SDF and IDF. Apart from that, the b-glucan content was analysed by using enzymatic kits, which were mixed-linkage beta glucan (Megazyme, Ireland), adopting the AACC method 32-23, AOAC method 995.16 and ICC Standard Method No. 168 (McCleary \& Glennie-Holmes, 1985), with a minor modification ( $0.1 \mathrm{~g}$ of sample used).

\subsection{Pasting properties}

The pasting properties of the biscuits were measured according to the method adopted from Mamat, Abu Hardan, and Hill (2010) and Sozer, Cicerelli, Heiniö, and Poutanen (2014), with some modifications. The biscuit sample in powder form $(3 \mathrm{~g}, \quad 1 \%$ moisture basis) was transferred into aluminium cups, and $25 \mathrm{ml}$ of distilled water was then added. Next, the suspension was placed into a rapid visco analyser, RVA (Newport Scientific, Australia), and the operating procedures were set accordingly. The suspension was heated to $50^{\circ} \mathrm{C}$, stirred at $160 \mathrm{rpm}$ for $10 \mathrm{~s}$ and then held at $50{ }^{\circ} \mathrm{C}$ for $1 \mathrm{~min}$. Subsequently, it was heated to $95^{\circ} \mathrm{C}$ in $3 \mathrm{~min}$ $42 \mathrm{~s}$ and the temperature continued to hold at $\quad 95^{\circ} \mathrm{C}$ for $2 \mathrm{~min}$ $30 \mathrm{~s}$. After that, the gelled sample was cooled to $50^{\circ} \mathrm{C}$ in $3 \mathrm{~min}$ $48 \mathrm{~s}$ and held at $50^{\circ} \mathrm{C}$ for $2 \mathrm{~min}$. The RVA gives information on the rheological properties of starch like viscosity. The peak viscosity (maximum viscosity during heating and holding at $95^{\circ} \mathrm{C}$ ), setback (the drop in apparent viscosity from trough viscosity), final viscosity (viscosity at the ending stage of the testing profile) and breakdown viscosity (hot paste) were identified from the pasting curve by utilising the Thermocline v. 2.2 software.

\subsection{Thermal characteristics}

The thermal characteristics (starch gelatinisation characteristics) of the samples were measured based on the method adopted from Mamat et al. (2010), with some modifications. The Pyris 6 Differential Scanning Calorimeter (DSC) (Perkin-Elmer Co., Norwalk, $\mathrm{CT}$ ) was used in this study. The samples were weighted (approximately $3.5 \mathrm{mg}$ ) in an aluminium DSC pan and moistened with distilled water, with the ratio of dry sample to water being approximately $1: 3$. The DSC pan was hermetically sealed and kept at room temperature for $12 \mathrm{~h}$ to reach an equilibrium condition. After that, the samples were scanned against a blank which was an empty DSC pan and heated at a rate of $\quad 5^{\circ} \mathrm{C} / \mathrm{min}$ from 5 to $95^{\circ} \mathrm{C}$. The starch gelatinisation parameters, generated by the DSC thermogram, were the onset temperature $\left(\begin{array}{ll}T^{\circ} & \end{array}\right)$, the peak or melting temperature $\left(T_{p}\right)$, the conclusion temperature $\left(T_{c}\right)$ and the enthalpy value $(\mathrm{DH})$. 


\subsection{Microstructure analysis}

In order to observe the starch granule morphology in all formulations of biscuits, they were trimmed into small fine pieces with a blade to produce a clean fracture surface and then viewed by using a scanning electron microscope (SEM) (brand: Fei, model: Quanta FEG 450, Netherland). The samples were mounted on an aluminium sample holder having a diameter of $12 \mathrm{~mm}$ with doublesided conductive carbon tape to improve conductivity. Then, the samples were sputter coated with a thin layer of gold in a vacuum evaporator (Baltex SCD005 Sputter Coater, Hi-Tech Germany) and located in the SEM chamber for investigation. The microstructures of the samples with magnifications of 1000 were photographed using $5 \mathrm{kV}$ of electron beam-accelerating voltage, and the diameters of the starch granules were measured.

\subsection{In vitro starch digestibility}

Starch digestibility in vitro was investigated according to the method adopted from Simsek and Nehir El (2015).

\subsubsection{Measurement of free glucose and total starch content}

Briefly, the samples $(3 \mathrm{~g})$ and $25 \mathrm{ml}$ acetate buffer $(0.1 \mathrm{M}, \mathrm{pH}$ $5.2)$ were mixed in a centrifuge tube $(50 \mathrm{ml}$ capacity) and incubated in a boiling water bath (Protech Model 830, Malaysia) for 30 min with frequent vortex mixing. The tubes were then cooled to $37^{\circ} \mathrm{C}$, and $0.2 \mathrm{ml}$ of invertase (Sigma-Aldrich, MO, USA) was added. This mixture was incubated in a shaking water bath (FOSS 1024 , Sweden) at $37^{\circ} \mathrm{C}$ for $30 \mathrm{~min}$. After the incubation, each sample $(1 \mathrm{ml})$ was pipetted into a test tube comprising $2 \mathrm{ml} \mathrm{96 \%}$ ethanol to determine the free sugar glucose (FSG) content by using a D-Glucose (glucose oxidase peroxidase: GOPOD) Assay Kit (Megazyme International K-GLUC, Ireland).

Alpha-amylase (Termamyl, $0.1 \mathrm{M}$ ) was added to the remainder of the solution. Then, the tubes were vortex mixed and incubated in a boiling water bath for $15 \mathrm{~min}$. Seven $\mathrm{M} \mathrm{KOH}(10 \mathrm{ml})$ were added to disperse the retrograded starch in a shaking water bath containing ice after the tubes were cooled in ice water. Each sample $(1 \mathrm{ml})$ was taken into $0.5 \mathrm{M}$ acetic acid $(10 \mathrm{ml})$, and $0.2 \mathrm{ml}$ of amyloglucosidase (Sigma-Aldrich, MO, USA) was added. The tubes were incubated in a shaking water bath at $60^{\circ} \mathrm{C}$ for $30 \mathrm{~min}$ and boiled for $10 \mathrm{~min}$ for enzyme inactivation, and $40 \mathrm{ml} \mathrm{66 \%}$ ethanol was added. The tubes were centrifuged at $10,000 \mathrm{~g}$ for $10 \mathrm{~min}$, and the amount of total glucose (TG) was measured by utilising the D-Glucose Assay Kit to determine the total starch (TS) content.

\section{TS ð\%P $1 / 4$ ठTG FSGP $0: 9$}

\subsubsection{Measurement of nutritionally important starch fractions}

Three grams of pancreatic enzyme (Sigma-Aldrich, MO, USA) was dispersed in water $(20 \mathrm{ml})$ for $10 \mathrm{~min}$ and centrifuged at $10,000 \mathrm{~g}$ for $10 \mathrm{~min}$. After the supernatant $(15 \mathrm{ml})$ was transferred to a beaker, $1.5 \mathrm{ml}$ of amyloglucosidase and $2 \mathrm{ml}$ of invertase were added to the solution. For each digestion, this enzyme solution was freshly prepared.

Samples ( $3 \mathrm{~g}$ ), $50 \mathrm{mg}$ of guar gum powder (Sigma-Aldrich, MO, USA) and $10 \mathrm{ml}$ of pepsin (50 mg pepsin (Sigma)/10 ml in $0.05 \mathrm{M}$ $\mathrm{HCl})$ were mixed in a test tube and were placed in a shaking water bath at $37{ }^{\circ} \mathrm{C}$ for $30 \mathrm{~min}$. Sodium acetate $(10 \mathrm{ml}, \quad 0.25 \mathrm{M})$ and five glass balls were then added to the test tube. After the addition of the above-prepared enzyme solution $(5 \mathrm{ml})$, the tubes were incubated horizontally in a shaking water bath at $37^{\circ} \mathrm{C}$. Aliquots $(0.5 \mathrm{ml})$ were taken at time intervals of $20 \mathrm{~min}$ and $120 \mathrm{~min}$, and they were mixed with $20 \mathrm{ml}$ of $66 \%$ ethanol. The hydrolysed glucose content was determined by the D-Glucose Assay Kit.

Nutritionally important starch fractions according to the rate of hydrolysis were calculated as rapidly digestible starch (digested within $20 \mathrm{~min}$ ) (RDS), slowly digestible starch (digested between 20 and $120 \mathrm{~min}$ ) (SDS) and resistant starch (undigested after $120 \mathrm{~min}$ ) (RS).

RDS ð\%॰ $1 / 4$ QGFSGP $0: 9$

SDS ð\%Р $1 / 4 \%$ \% G ${ }_{20}$ P $0: 9$

RSð\%P $1 / 4$ ठTG G $_{20}$ P $\quad 0: 9$

References for the formulas are as follow:

$\mathrm{G}_{20}=$ quantity of free glucose measured after 20 min incubation with enzyme.

$\mathrm{G}_{120}=$ quantity of free glucose measured after $120 \mathrm{~min}$ incubation with enzyme.

\subsection{In vivo $\mathrm{GI}$ determination}

\subsubsection{Experimental design}

The study was conducted using the internationally recognised GI methodology (FAO/WHO, 1998). Eleven healthy participants (four males and seven females with no histories of carbohydrate malabsorption and smoking; ages ranged from 20 to 65 years old; body mass index of $18.5-24.9 \mathrm{~kg} / \mathrm{m}^{2}$ ) were taken part in this study. This controlled crossover clinical trial involved seven experimental sessions in which participants were randomly assigned to consume glucose (Glucolin, Reckitt Benckiser, Malaysia) as reference food and test foods containing $25 \mathrm{~g}$ of available carbohydrate on separate occasions after overnight fasting. Glucose consumption was repeated three times by each participant to improve the measurement precision and hence reduce the variation of mean $\mathrm{GI}$ values due to glucose tolerance. After the fasting blood glucose readings were taken, the reference food and test foods were given with $250 \mathrm{ml}$ of water, and the participants were allowed to eat at a comfortable pace within $15 \mathrm{~min}$. A minimum of a 3-day gap was maintained as the washout period between the test meals to minimise any carryover effect. Ethical approval for this study was acquired from the Human Research Ethics Committee of Universiti Sains Malaysia (FWA Reg. No: 00007718; IRB Reg. No: 00004494), and all of the participants were given written informed consent.

\subsubsection{Blood glucose measurements}

Fasting blood glucose samples were taken before the test foods were given to the participants $(0 \mathrm{~min})$, and further blood samples were collected at $15,30,45,60,90$ and 120 min after consuming the test foods. Each time, a few drops of blood were obtained by a capillary finger prick, and the blood glucose was measured by using a dry chemistry analyser (Reflotron Plus, Roche, Switzerland), which applies the glucose oxidase-based method. According to $\mathrm{FAO} / \mathrm{WHO}$ (1998), capillary blood glucose is preferred over venous blood glucose because it is easier to get, the rise in blood glucose is greater and the results are less variable.

\subsubsection{Calculation of $\mathrm{Gl}$}

The calculation of the incremental area under curve (iAUC) was done using Microsoft Excel (Version 2007, USA), in which the trapezoid rule was used. All areas below the baseline were omitted from the calculations. The GI, defined as the iAUC for each test food, was expressed as a percentage of the mean iAUC for glucose consumed by the same participant. The GI was determined from the iAUCs with each participant acting as his/her own rference. 
The resulting values for all participants were averaged to calculate the $\mathrm{Gl}$ for each test food.

$$
\begin{aligned}
& \text { GI } 1 / 4 \frac{\text { Area under the blood glucose curve of test food }}{\text { Area under the blood glucose curve of reference foodðglucosep }} \\
& 100
\end{aligned}
$$

\subsection{Sensory evaluation}

The sensory evaluation of biscuits was accomplished by 60 consumers consisting of staff members and students of the School of Health Sciences, Universiti Sains Malaysia. Consumers received four different formulations of biscuits for sensory test. The tested samples were coded with three-digit numbers and randomly permutated. All samples were evaluated according to the sevenhedonic scaling method (Aminah \& Tan, 2001). The sensory parameters evaluated were aroma, colour, appearance, crispiness, flavour and overall acceptance on a seven-point scale (1 = dislike the most, and $7=$ like the most).

\subsection{Statistical analysis}

Data obtained were subjected to one-way analysis of variance (ANOVA) followed by Bonferroni's multiple comparisons test to compare the mean differences among the samples. The statistical analysis was done by using GraphPad Prism version 6.0 for windows (GraphPad Software, San Diego California USA). Results were expressed as mean \pm standard deviation (SD). All measurements were taken in triplicate $(n=3) \quad$ except DF compositions $(n=4)$. Each experiment was repeated three times. Significance level was set at $\mathrm{P}<0.05$.

\section{Results and discussions}

\subsection{Nutritional values}

Proximate analyses indicated that the PSC powder contained a low level of moisture $(7.04 \%)$ and fat $(2.30 \%)$ but a high amount of ash $(7.79 \%)$, protein $(22.41 \%)$ and carbohydrate $(60.47 \%)$, resulting in a $451.60 \mathrm{kcal} / 100 \mathrm{~g}$ calorific value. Furthermore, PSC powder possesses negligible amount of sucrose at the level of $0.19 \%$, which is widely acceptable for a population, especially diabetic individuals who are concerned about their blood glucose levels and health statuses. Interestingly, appreciable amounts of TDF (56.99\%), IDF $(48.79 \%)$, SDF $(8.21 \%)$ and b-glucan $(3.32 \%)$ were also detected in the PSC powder, as DF is abundant in the cell walls of mushroom

\begin{tabular}{|c|c|c|c|c|}
\hline \multirow[t]{2}{*}{ Nutritional compositions } & \multicolumn{4}{|l|}{ Concentration (\%) } \\
\hline & Control (0\% PSC) & $4 \%$ PSC & $8 \%$ PSC & $12 \%$ PSC \\
\hline Protein & $6.50 \pm 0.06$ & $6.91 \pm 0.18^{a}$ & $7.43 \pm 0.22^{a b}$ & $7.85 \pm 0.17^{a b c}$ \\
\hline Ash & $0.86 \pm 0.02$ & $0.88 \pm 0.04$ & $0.95 \pm 0.08^{a b}$ & $1.00 \pm 0.10^{a b c}$ \\
\hline Fat & $22.68 \pm 0.06$ & $22.75 \pm 0.34$ & $22.90 \pm 0.26$ & $23.08 \pm 0.46$ \\
\hline Moisture & $1.37 \pm 0.05$ & $1.34 \pm 0.09$ & $1.25 \pm 0.13^{a b}$ & $1.16 \pm 0.15^{\mathrm{abc}}$ \\
\hline Carbohydrate & $68.59 \pm 0.08$ & $68.20 \pm 0.54$ & $67.47 \pm 0.24$ & $66.92 \pm 0.96^{a}$ \\
\hline Calorific value (kcal/100 g) & $499.03 \pm 1.95$ & $499.36 \pm 2.75$ & $499.49 \pm 3.04$ & $499.48 \pm 2.62$ \\
\hline Sucrose & $12.80 \pm 0.06$ & $12.80 \pm 0.41$ & $12.79 \pm 0.26$ & $12.81 \pm 0.19$ \\
\hline TDF & $3.37 \pm 0.19$ & $6.19 \pm 0.06^{a}$ & $8.62 \pm 0.14^{a b}$ & $9.84 \pm 0.12^{a b c}$ \\
\hline IDF & $2.63 \pm 0.16$ & $4.39 \pm 0.17^{a}$ & $6.53 \pm 0.19^{a b}$ & $7.46 \pm 0.15^{\mathrm{abc}}$ \\
\hline SDF & $0.74 \pm 0.04$ & $1.80 \pm 0.27^{a}$ & $2.10 \pm 0.10^{a b}$ & $2.38 \pm 0.12^{a b c}$ \\
\hline b-glucan & $0.12 \pm 0.01$ & $1.06 \pm 0.03^{a}$ & $1.29 \pm 0.04^{\mathrm{ab}}$ & $1.78 \pm 0.09^{a b c}$ \\
\hline
\end{tabular}

Table 1

Nutritional compositions of biscuits formulated with PSC powder (mean \pm SD). fruiting bodies. The nutritional compositions of biscuits formulated with PSC powder as a partial replacement for wheat flour at four different levels are presented in Table $1 . \quad$ Basically, increasing the level of the PSC powder from 0 to $12 \%$ resulted in a significant $(P<0.05)$ increase in the level of protein, ash, TDF (including SDF and IDF) and b-glucan, with the $12 \%$ PSC biscuit recorded the highest nutritional values.

Collectively, the DF compositions of biscuits showed that PSC powder could be used for enriching the DF content of biscuits. is due to the fact that PSC powder originally contains high level of TDF, including its soluble form and insoluble form. To claim that a food is a 'source of DF', the food should contain at least $3 \mathrm{~g}$ of DF per $100 \mathrm{~g}$ of serving. Meanwhile, to claim that a food is 'high in DF', the product must contain at least $6 \mathrm{~g}$ of DF per $100 \mathrm{~g}$ of serving (Foschia, Peressini, Sensidoni, \& Brennan, 2013). Therefore, PSCenriched biscuits containing TDF of $6.19-9.84 \%$ are considered to be high-DF products. The same trends of increased DF content were reported in biscuits formulated with increasing levels of cinnamon powder ( $\mathrm{Ng} \&$ Wan Rosli, 2014). Due to the universal consumption of bakery products such as biscuit, enriching biscuit with DF offers promising potential to increase populations' daily DF intake. Apart from that, PSC powder addition also increased the nal high amounts of protein and ash in PSC powder.

However, there was an inverse relationship between moisture content and the incorporation level of PSC powder in the biscuit formulations. The moisture content of the biscuits was shown to decrease significantly $(P<0.05)$ at 8 and $12 \%$ PSC powder incorporation as compared to the control biscuit. It is because the DF contents in PSC powder may absorb a large amount of water, eventually resulting in a decrease in the moisture content. The findings were in consistent with Ayo, Ayo, Nkama, and Adewori (2007), who incorporated soybean flour to partially replace wheat flour in biscuits. Biscuits are considered very-low-moisturecontent products, as thermal processing brings down the final moisture content (ranged from 1 to $5 \%$ ) in the product. The moisture content of a biscuit is basically used as an indicator of biscuit quality. It is essential to measure it because of its potential impact on the sensory, textural and microbial properties of final products. This low moisture content ascertains that biscuits are basically free from microbiological spoilage and thus confers a long shelf life on the products.

\subsection{Pasting properties}

The pasting properties of biscuit were obtained to understand its functional behaviour during cooling and heating periods, which protein and ash content of biscuits, mostly attributed to the origi-

TDF, total dietary fibre; IDF, insoluble dietary fibre; SDF, soluble dietary fibre.

a $\mathrm{P}<0.05$ as compared to control (0\% PSC) biscuit.

b $\mathrm{P}<0.05$ as compared to $4 \% \mathrm{PSC}$ biscuit.

c $\mathrm{P}<0.05$ as compared to $8 \%$ PSC biscuit. 
is typical during the processing of starchy products or foods where wheat flour (starch) is added as food ingredient. According to Adebowale, Adegoke, Sanni, Adegunwa, and Fetuga (2012), peak viscosity is an index of the ability of starch-based foods to swell freely prior to their physical break down. High peak viscosity is attributed to the high starch content of food. Final viscosity expresses the capacity of the material to develop a viscous paste. Setback viscosity is the indication of syneresis or retrogradation tendency of starch upon cooling of cooked starch pastes whereas breakdown viscosity is the indication whether the swollen starch granules can be disintegrated. In general, with increasing the incorporation levels of PSC powder in biscuits, all of the pasting viscosities were shown to decrease in a dose-related response (Table 2). Compared with the control biscuit, there were significant $(P<0.05)$ reductions for all the pasting viscosities for the biscuits enriched with 8 and $12 \%$ PSC powder, while there were no significant differences for the biscuit enriched with $4 \%$ PSC powder. The decrease in all the pasting viscosities with increasing the incorporation levels of DF source was also seen in the work by Sozer et al. (2014), when 5, 15 and $30 \%$ wheat bran was added to flour-bran mixtures.

When the starch granules composed of amylose and amylopectin were heated in excess water, the branched linkages at a-1,6 location on amylopectin were easily broken down. The amylopectin absorbed more water, making the starch granules swelled to 10-20 times their original sizes. As an outcome of this swelling, the granules burst open, and the amylose leached out of the swollen starch granules, causing loss of order and crystallinity which resulted in an increase in the viscosity of starch suspension (BeMiller, 2011). However, by increasing the incorporation level of DF-rich PSC powder which had high water holding capacity $(13.46 \mathrm{~g} / \mathrm{g})$, it competed with starch for water and thus absorbed more water than the starch (wheat flour). This situation resulted in the reduction of the starch's strength of absorbing water and hence leading to a decrease in all the pasting viscosities. Moreover, the lower pasting viscosities are proposed to be an indication of reduced starch accessibility for gelatinisation after the partial supplementation of wheat flour with the DF sources (Collar, Santos, \& Rosell, 2006).

On the other hand, the pasting temperatures of the biscuits increased as the PSC powder addition level increased. In comparison to the control biscuit, there were significant $(P<0.05)$ increments for the pasting temperatures for the biscuits enriched with 8 and $12 \%$ PSC powder, while there was no significant difference for the pasting temperature for the biscuit enriched with $4 \%$ PSC powder. Pasting temperature provides an indication of the minimum temperature required to cook the sample. Higher pasting temperatures might be linked to restricted or delayed starch swelling and amylose leaching (Mira, Eliasson, \& Persson, 2005).
Increasing wheat bran supplementation has also previously been shown to reduce pasting viscosities and increase pasting temperatures (Sozer et al., 2014).

\subsection{Thermal characteristics}

When heated in the existence of sufficient water, starch undergoes an order-disorder phase transition in which the intermolecular bonds of starch molecules are broken down. This situation allows the exposed hydroxyl groups to engage water molecules and the process is defined as starch gelatinisation. Native starch granules are only vulnerable to enzymes in the surface layers, while gelatinisation destroys the starch granule structure, enlarges the surface area and thereby increases the starch's vulnerability to enzymes (Schuchardt et al., 2016). The most usually used technique in the studies of starch gelatinisation is DSC, which explains an endothermic event at the temperature similar to those at which structural alterations are observed. The event is usually equated with the thermal melting of crystalline. DSC measures the enthalpy of gelatinisation and gelatinisation transition temperatures which are affected by the molecular architecture or degree of crystallinity. The thermal properties of the biscuits formulated with PSC powder are shown in Table 2.

With the increasing incorporation levels of PSC powder, the gelatinisation transition temperatures $\left(T \quad o, T_{p}\right.$ and $\left.T_{c}\right)$ were shown to increase. Basically, the gelatinisation temperatures were significantly higher at $12 \%$ PSC powder incorporation, indicating that it required higher temperature to melt its crystalline structure. As the content of DF increased with the increasing levels of PSC powder, it might have been competing with starch for water absorption, thus limiting starch-swelling and gelation events and hence contributing to a higher starch gelatinisation temperature. DF hindered starch gelatinisation as majority of the starch remained intact after thermal treatment. A similar finding has been documented in pasta containing pea fibre (Tudorica, Kuri, \& Brennan, 2002).

Apart from that, the enthalpy value ( $\mathrm{DH}$ ) was significantly $(P<0.05)$ decreased with the increasing incorporation levels of PSC powder. Higher enthalpy of gelatinisation indicates an overall measure of crystallinity (quantity and quality) and is an index of the loss of the molecular order within the starch granule (BeMiller, 2011). Food enriched with DF was previously shown to increase the food's water-holding capacity, making an insufficient amount of water available for the gelatinisation of starch. Furthermore, the substitution of wheat flour (source of starch) with PSC powder in the biscuit formulations resulted in the reduction of available starch for gelatinisation, which, in turn, reduced the enthalpy values. The result was supported by Sozer et al. (2014), who employed wheat bran as a DF source in biscuit formulations.

Table 2

Pasting and thermal properties of biscuits formulated with PSC powder (mean \pm SD).

\begin{tabular}{|c|c|c|c|c|}
\hline Properties & Control (0\% PSC) & $4 \% \mathrm{PSC}$ & $8 \%$ PSC & $12 \%$ PSC \\
\hline Peak viscosity (cP) & $314.33 \pm 4.52$ & $307.00 \pm 6.07$ & $297.67 \pm 3.48{ }^{a b}$ & $291.67 \pm 3.96{ }^{a b}$ \\
\hline Breakdown viscosity (cP) & $111.00 \pm 3.20$ & $108.33 \pm 2.58$ & $102.33 \pm 1.98^{a}$ & $97.67 \pm 2.03^{a b}$ \\
\hline Final viscosity $(\mathrm{cP})$ & $488.67 \pm 5.66$ & $482.00 \pm 4.95$ & $474.67 \pm 6.12^{a}$ & $470.00 \pm 6.23^{a b}$ \\
\hline Setback viscosity (cP) & $286.33 \pm 2.28$ & $284.33 \pm 4.56$ & $279.00 \pm 5.33^{a}$ & $276.67 \pm 5.87^{\mathrm{ab}}$ \\
\hline Pasting temperature $\left({ }^{\circ} \mathrm{C}\right)$ & $83.77 \pm 6.50$ & $84.24 \pm 4.32$ & $87.05 \pm 7.82^{a b}$ & $88.85 \pm 3.70^{a b}$ \\
\hline $\mathrm{T}_{\mathrm{o}}\left({ }^{\circ} \mathrm{C}\right)$ & $58.44 \pm 1.47$ & $58.71 \pm 2.03$ & $58.80 \pm 2.45$ & $59.05 \pm 1.87^{a}$ \\
\hline $\mathrm{T}_{\mathrm{p}}\left({ }^{\circ} \mathrm{C}\right)$ & $60.97 \pm 1.14$ & $61.15 \pm 2.58$ & $61.66 \pm 2.39$ & $61.86 \pm 1.56^{a}$ \\
\hline $\mathrm{T}_{\mathrm{c}}\left({ }^{\circ} \mathrm{C}\right)$ & $62.54 \pm 2.02$ & $62.98 \pm 2.97$ & $63.43 \pm 3.10^{a}$ & $64.05 \pm 1.82^{\mathrm{ab}}$ \\
\hline $\mathrm{DH}(\mathrm{J} / \mathrm{g})$ & $2.33 \pm 0.13$ & $2.29 \pm 0.06^{a}$ & $2.10 \pm 0.09^{a b}$ & $2.03 \pm 0.10^{a b c}$ \\
\hline
\end{tabular}

$T_{0}$, onset temperature; $T_{p}$, peak temperature; $T_{c}$, conclusion temperature; $D H$, enthalpy value.

a $\mathrm{P}<0.05$ as compared to control $(0 \% \mathrm{PSC})$ biscuit.

b $\mathrm{P}<0.05$ as compared to $4 \% \mathrm{PSC}$ biscuit.

${ }^{c} \mathrm{P}<0.05$ as compared to $8 \%$ PSC biscuit. 
In addition, the reduction in the enthalpy of starch gelatinisation could be associated with reduced pasting properties as observed earlier in a PSC-enriched biscuit.

\subsection{Microstructures}

The microstructures of all formulations of the biscuits were viewed by using SEM to observe the characteristics of the starch granules. Visual magnifications of 1000 of four different formulations of biscuits are shown in Fig. 1. Starch granules were randomly selected, and the measurement of the diameter was done by using SEM instrument scales. By observation, SEM micrographs showed that the diameters of the starch granules were reduced directly proportional to the PSC powder added. For the control (Fig. 1A) and 4\% PSC biscuit (Fig. 1B), both of the starch granules were observed to retain their granular identity even they are elongated and appear to be fused with neighbouring granules as constituent of a continuous network. Moreover, they are featured by an open structure with a larger starch-granule diameter, corresponding to the entrapment of gas bubbles that expand during baking, which might greatly enhances the surface area exposed to enzyme activity. A similar observation was found based on a research study examining the effect of DF on the internal structures of pastas (Brennan \& Tudorica, 2008).

Nevertheless, the DF-rich 12\% PSC biscuit (Fig. 1D) showed the smallest starch granules' diameters ranging from 15.91 to $19.25 \mathrm{I} \mathrm{m}$. This is attributed to the entrapment of starch granules within the fibre matrix. In addition, it could be noticed that the DF component of PSC powder embedded in the biscuits disturbed the starch granule structures, making them irregularly shaped and feature less smooth granule surfaces. Hence, DF reduced the retention of the integrity of the starch granules by reducing the diameters of starch granules and disturbing the structures the starch granules, both of which resulted in restricted starch susceptibility to enzymatic hydrolysis (Wu,

Chiou, Weng, Yu, \& Wang, 2014).

The SEM result supports the RVA profiles and DSC measurements, which reported the reduced pasting viscosities and the reduced starch gelatinisation properties of PSC-enriched biscuits, respectively. The smaller starch granular sizes bind less water and thus reduce the pasting viscosities and the starch gelatinisation. Starch granules in the PSC-enriched biscuits were only partially gelatinised due to the presence of DF and the lack of sufficient water. This was in agreement with the previous study done on rich tea biscuits (Mamat et al., 2010).

\subsection{Starch digestibility}

Starch is constituted by glucose polymers connected together by the $a-1,4$ and $a-1,6$ glycosidic bonds in the form of amylose and amylopectin, which could be hydrolysed by amylase and debranching enzyme. Biscuit products primarily consist of starch, which explains why the determination of the physiological functionality of the starch becomes very crucial. The effects of PSC powder addition on the in vitro starch digestibility of biscuits were examined in this study. As shown in Table 3, the obtained results generally showed significant differences in comparison to the control (0\% PSC powder) biscuit regarding the contents of different starch fractions (RDS, SDS and RS),TS, RAG (rapidly available glucose), SAG (slowly available glucose) and the starch digestion rate index (SDRI).

The wheat flour consisted mainly of starch. As increasing levels of PSC powder were used to replace wheat flour in the biscuit formulations, there was a reduced content of TS in the biscuits, taking into account that the biscuits formulating with PSC powder had initial starch content lower than that of the control biscuit. The TS content in the control biscuit was the highest (39.21\%), while that of the biscuit enriched with $12 \%$ PSC powder was the lowest
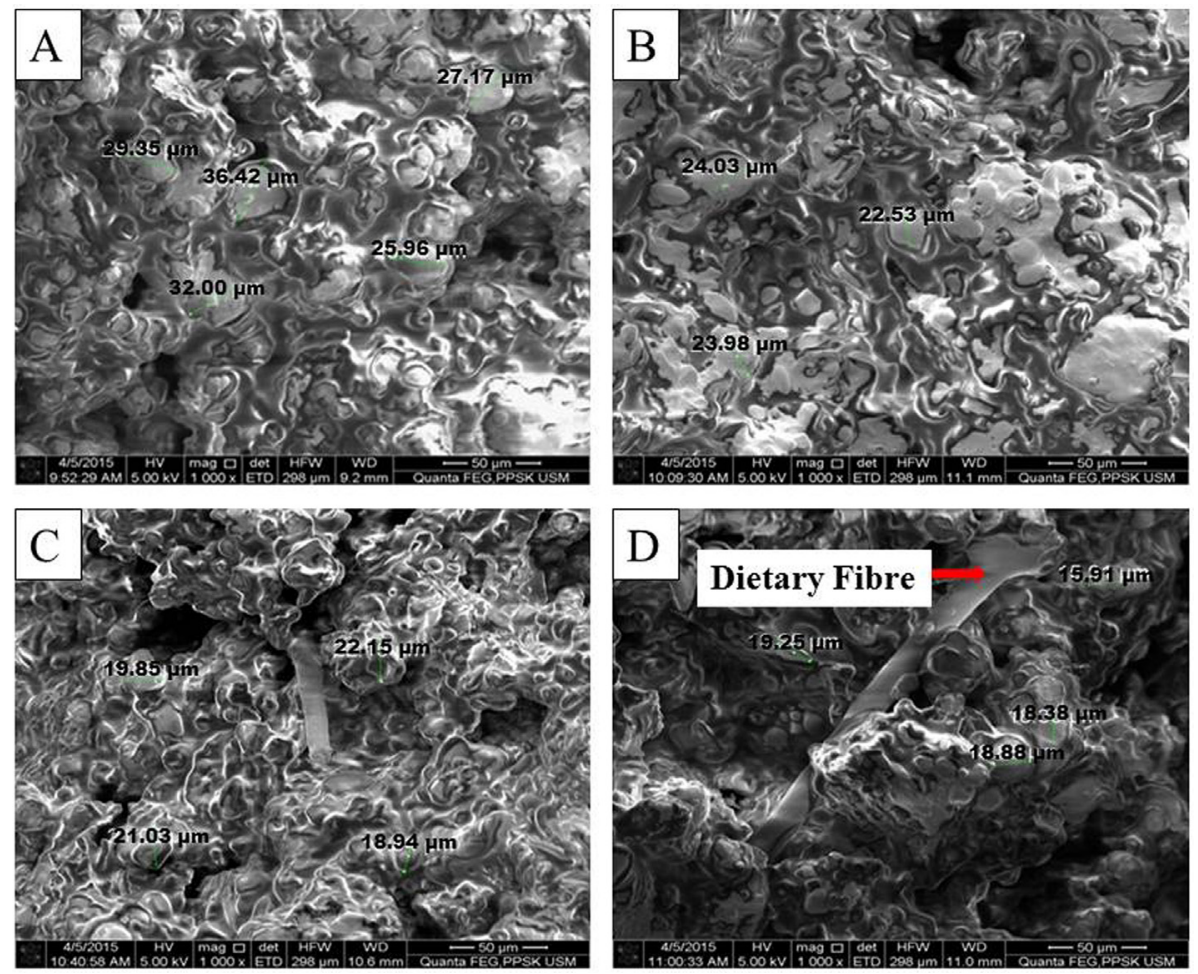

Fig. 1. Scanning electron micrographs (magnification of 1000) of control biscuit (A), biscuit with 4\% PSC powder (B), PSC powder (D). 
Table 3

Starch digestibility of biscuits formulated with PSC powder determined by in vitro enzymatic hydrolysis (mean \pm SD).

\begin{tabular}{|c|c|c|c|c|}
\hline \multirow[t]{2}{*}{ Properties } & \multicolumn{4}{|l|}{ Percentage (\%) } \\
\hline & Control (0\% PSC) & $4 \%$ PSC & $8 \%$ PSC & $12 \% \mathrm{PSC}$ \\
\hline TS & $39.21 \pm 1.05$ & $37.40 \pm 0.84$ & $35.81 \pm 0.94^{\mathrm{ab}}$ & $33.94 \pm 1.67^{a b}$ \\
\hline RDS & $33.84 \pm 0.78$ & $28.62 \pm 0.89^{a}$ & $23.72 \pm 0.33^{a b}$ & $20.36 \pm 0.32^{a b c}$ \\
\hline SDS & $3.15 \pm 0.12$ & $5.19 \pm 0.11^{a}$ & $8.36 \pm 0.18^{a b}$ & $10.00 \pm 0.23^{a b c}$ \\
\hline RS & $3.24 \pm 0.46$ & $3.93 \pm 0.20$ & $4.65 \pm 0.12^{a b}$ & $4.93 \pm 0.11^{\mathrm{ab}}$ \\
\hline RAG & $46.1 \pm 1.37$ & $40.58 \pm 0.56^{a}$ & $35.35 \pm 1.25^{a b}$ & $31.82 \pm 1.33^{a b c}$ \\
\hline SAG & $12.05 \pm 0.32$ & $14.54 \pm 0.32^{a}$ & $18.33 \pm 0.55^{a b}$ & $20.42 \pm 0.28^{a b c}$ \\
\hline SDRI & $87.55 \pm 3.38$ & $75.91 \pm 2.91^{a}$ & $66.45 \pm 2.46^{\mathrm{ab}}$ & $60.32 \pm 2.19^{a b c}$ \\
\hline
\end{tabular}

TS, total starch; RDS, rapidly digestible starch; SDS, slowly digestible starch; RS, resistant starch; RAG, rapidly available glucose; SAG digestion rate index.

a $\mathrm{P}<0.05$ as compared to control ( $0 \%$ PSC) biscuit.

b $\mathrm{P}<0.05$ as compared to $4 \% \mathrm{PSC}$ biscuit.

${ }^{c} \mathrm{P}<0.05$ as compared to $8 \% \mathrm{PSC}$ biscuit.

(33.94\%). Depending on the extent and rate of in vitro digestion, starch can be classified into three major categories. The first two (SDS and RDS) fractions are completely digested in the small intestine with different rates meanwhile the RS as third category is not enzymatically degraded in the small intestine but fermented in the large intestine (Giuberti, Gallo, Cerioli, Fortunati, \& Masoero, 2015). In present study, it can be seen that the content of RDS decreased $(P<0.05)$ significantly, but SDS and RS increased significantly $(P<0.05)$, with increasing levels of PSC powder added.

The SDRI is RDS expressed as a percentage of TS in the food. It is an indicator of the susceptibility of starch to hydrolysis. In this study, the SDRI for the biscuit incorporated with $12 \%$ PSC powder recorded the lowest, indicating that the biscuit was more difficult to be hydrolysed. Other useful indexes for the assessment of the digestibility of starch are the RAG and SAG values. RAG is the amount of free glucose measured after a given food is incubated with digestive enzymes for $20 \mathrm{~min}\left(\mathrm{G}_{20}=\mathrm{RAG}\right)$. SAG is the amount of free glucose measured after 120 min of incubation minus that measured at 20 min $\left(G_{120} G_{20}=S A G\right)$. The partial substitution of wheat flour with PSC powder in biscuits resulted in a significant high level of SAG value and a low level of RAG value, reflecting a slow release of glucose from the starch. Since the RAG value includes both free glucose and RDS, it was revealed to be a good indicator for the blood glucose and insulin response of test foods (Englyst, Vinoy, Englyst, \& Lang, 2003).

Several factors can explain the marked differences in starch digestibility. The lower digestibility of biscuits enriched with increasing levels of PSC powder might be attributed to the higher DF content. DF reduced starch digestibility in various different ways. Its water-holding capacity makes less water for starch swelling and starch gelatinisation as shown in Section 3.3. Similar research was done by Vujic ', Vitali Čepo, and Vedrina Dragojevic (2015), who demonstrated that soy and amaranth addition decreased the rate of the starch digestibility of a biscuit. Other than that, DF disturbed the structures of the starch granules and reduced the diameters of the starch granules (explained in Section 3.4), both of which resulted in reducing the surface exposed to enzyme activity and, in turn, reducing starch digestibility.

In addition, protein plays an essential role in reducing starch digestibility. The protein content of the biscuits increased directly proportional to the increasing levels of PSC powder added due to the original high protein content of the PSC powder (22.41\%). Protein reduced the starch granule surface accessibility and hence affected the enzyme susceptibility to hydrolysing starch into glucose. More specifically, protein fractions, such as globulin and albumin, were glued into a matrix surrounding starch granules acting as a barrier against starch digestion. This phenomenon has been confirmed by removing gluten from wheat flour or by adding protease to corn flour, both of which resulted in a marked enhancement of in vitro starch digestibility (Singh, Dartois, \& Kaur, 2010).

Besides nutritional elements, such as DF and protein, the reduction of RDS content and increase of SDS content can be explained by the amorphous and crystalline structures of starch. The reduced starch digestibility was associated with the reduced starch gelatinisation resulting from the addition of the PSC powder. Basically, starch gelatinisation was featured by physical changes of starch, such as the rupturing, swelling and disruption of a crystalline structure. Native starch was hydrolysed slowly compared to the gelatinised starch in which the crystallinity and molecular order have been lost and where the accessibility of substrate to enzyme is greater. Ren et al. (2016) suggested that in terms of biochemical changes, the starch gelatinisation was such a process that RS and SDS turned into RDS. Hence, reduced starch gelatinisation enthalpy value limited the turning process.

Based on these results, it could be concluded that the PSCenriched biscuit was markedly more resistant to digestion compared to the control biscuit without the addition of PSC powder. Since both of the lower RDS content and higher SDS content are directly correlated with reduction of postprandial glycaemic response (Vujic' et al., 2015), the addition of PSC powder represents a convenient and novel choice in lowering the $\mathrm{Gl}$ of the final product.

\subsection{Glycaemic index}

To provide a comprehensive evaluation of the effects of PSC powder addition on the starch digestibility of biscuits, the blood glucose response after the ingestion of PSC-enriched biscuits was further investigated and presented in Fig. 2A. The peak time and concentration are two main factors of the blood glucose curve. After $15 \mathrm{~min}$, the blood glucose levels of all four test foods (ranged from $5.5 \pm 0.3$ to $6.0 \pm 0.6$, mean $\pm S D$ ) were recorded significantly lower $(P<0.05)$ in comparison to the reference food $(7.0 \pm 0.5)$. The peak time of all test foods was $30 \mathrm{~min}$. It can be observed that the peak concentration of the reference food at $30 \mathrm{~min}$ was the highest $(8.3 \pm 0.4)$, followed by the control biscuit $(7.0 \pm 0.7), 4 \%$ PSC biscuit $(6.7 \pm 0.3), 8 \%$ PSC biscuit $(6.6 \pm 0.5)$ and $12 \%$ PSC biscuit $(6.0 \pm 0.3)$. The blood glucose levels of all four test foods at $30 \mathrm{~min}$ were documented to have significant lower $(P<0.05)$ compared to the reference food; also, the $12 \%$ PSC biscuit showed a significantly $(P<0.05)$ lower blood glucose level peak compared to those of all test foods.

The calculation of iAUC (Fig. 2B) for each participant and each food allowed for the evaluation of the GI of the test foods (Fig. 2 C). The iAUC for glucose was the highest $(167.9 \pm 7.4)$, followed by that of the control biscuit $(96.1 \pm 8.1), 4 \%$ PSC biscuit 

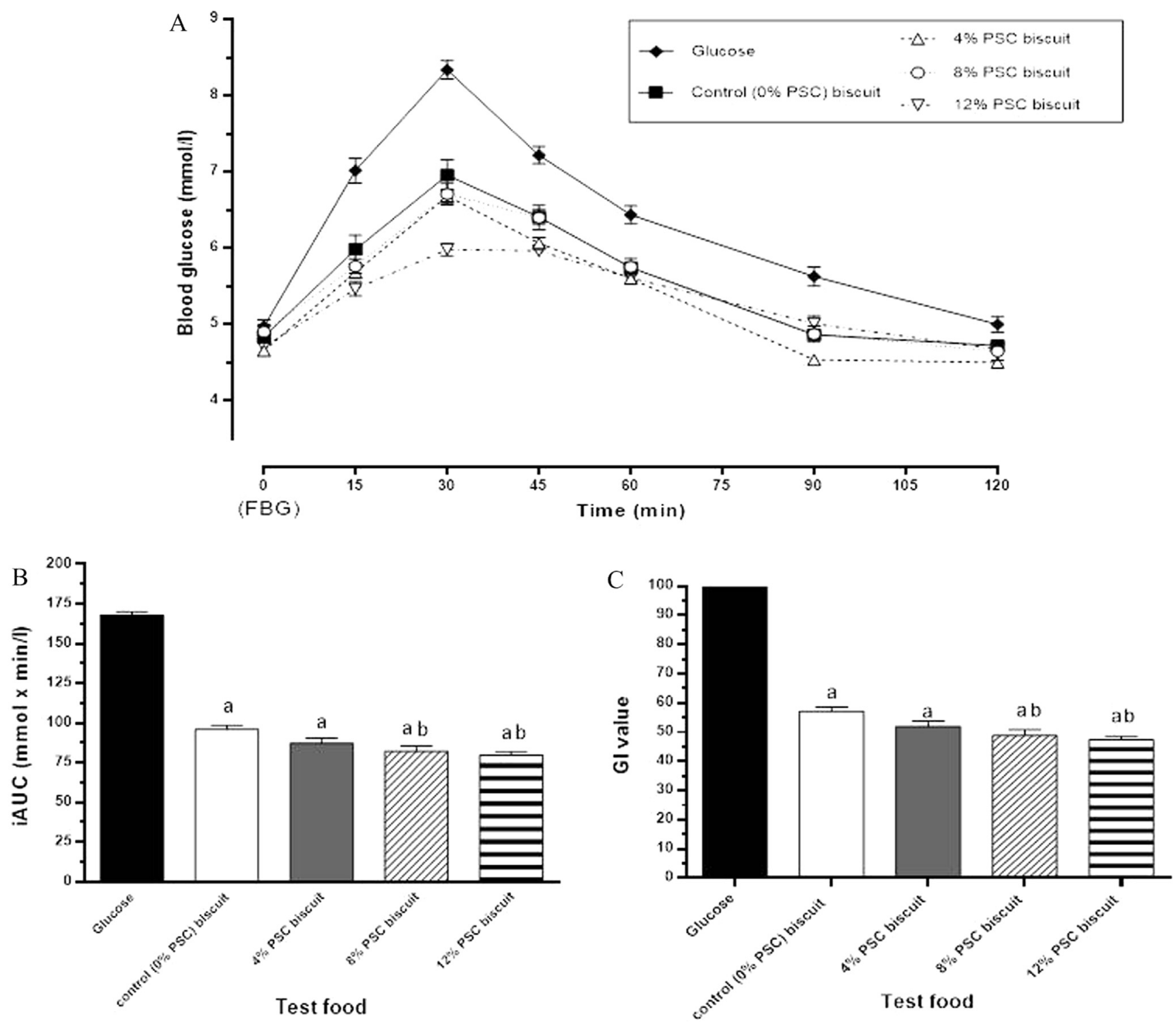

Fig. 2. Mean blood glucose response (A), iAUC (B) and GI value (C) of glucose as reference food and four formulations of biscuits incorporated with $0,4,8$ and $12 \%$ PSC powder. ${ }^{\mathrm{a}} \mathrm{P}<0.05$ vs. glucose; ${ }^{\mathrm{b}} \mathrm{P}<0.05$ vs. control biscuit $(0 \% \mathrm{PSC})$.

(87.3 \pm 10.4$), 8 \%$ PSC biscuit $(82.3 \pm 11.0)$ and $12 \%$ PSC biscuit (79.7 \pm 7.4$)$. Apparently, all test foods (biscuits enriched with 0,4 , 8 and $12 \%$ PSC powder) were detected to have significant $(P<0.05)$ differences in the mean value of iAUC in comparison to the reference food. Apart from that, the iAUC for the 8 and $12 \%$ PSC biscuit appeared to be markedly $(P<0.05)$ lower compared to that of the reference food and the control biscuit, while there was no significant difference detected between the iAUC for the $4 \%$ PSC biscuit and control biscuit.

The $\mathrm{GI}$ is the ranking (on a scale of $0-100$ ) of different dietary carbohydrate-rich foods, depending on how fast and how high the blood glucose rises, and how quickly the body responds by bringing it back to normal after food ingestion (Jenkins et al., 2008). The food of medium $\mathrm{Gl}$ is between 56 and 69.5, whilst the food of low $\mathrm{Gl}$ is regarded as being 55 or less (Schuchardt et al.,

2016). The calculated $\mathrm{GI}$ for the control biscuit ( $\mathrm{GI}=57.2 \pm 4.8)$ was concordant with the levels deemed to establish a mediumGI food, whereas the 4, 8 and $12 \%$ PSC biscuits could be considered as low-Gl foods, with the $\mathrm{Gl}$ values recorded as $52.0 \pm 6.2$, $49.0 \pm 6.5$ and $47.4 \pm 4.4$, respectively. Among all PSC-enriched bis- cuits, the GI for the 8 and $12 \%$ PSC biscuits were markedly lower as compared to that of the control biscuit.

DF performs a decisive role in formulating a low-GI biscuit. DF is a non-starch polysaccharide featured by more than 10 degrees of polymerisation and the chemical bonds between the D-glucose monomeric units are mainly b-glycosidic linkages. IDF and SDF which are distinguished by their water solubility have different mechanistic effects in reducing glycaemic response. For PSCenriched biscuit as starch-containing food, the GI mainly depends on the starch granules' accessibility to digestive enzymes. IDF, a water-insoluble polymer, acts as a physical barrier and entraps the starch granules within its fibrous network, contributing to the loss of starch granule integrity by reducing the diameter and altering the shape of starch granules. Subsequently, starch molecules of biscuit are less susceptible to enzymatic hydrolysis by a-amylase under human digestion, and thus the conversion rate of starch to glucose become slower (Vujic ' et al., 2015). Beside the restriction of starch digestion, IDF delays intestinal glucose absorption by retarding glucose diffusion across the intestinal membrane and, in turn, reducing postprandial glycaemic response and $\mathrm{GI}$ of biscuit. 
The mechanisms of action behind IDF are not limited to starch digestion and glucose absorption. Lattimer and Haub (2010) reported that ingestion of IDF stimulates an accelerated secretion of glucose-dependent insulinotropic polypeptide (GIP). GIP, an incretin hormone, triggers postprandial insulin release which promotes the uptake of glucose from the blood to fat, liver and skeletal muscle cells. Furthermore, short chain fatty acids which are produced by bacteria in the gut during fermentation of IDF have been demonstrated to reduce postprandial glucose response. An increase in free fatty acids in the blood can inhibit glucose metabolism through the inhibition of GLUT4 transporters. Hence, short chain fatty acids, by way of reducing free fatty acids in the blood, can also decrease postprandial blood glucose levels and GI through the competition in insulin-sensitive tissues.

Apart from that, the effectiveness of PSC-enriched biscuit in reducing the postprandial rise in blood glucose level has been attributed to the presence of (1 ? 3, 1? 4)-b- D-glucan, a type of SDF giving viscous solutions. The mechanism by which b-glucan decreases postprandial glycaemic response is associated with the effect of its viscosity on the gastrointestinal event, which lowers the starch granules' vulnerability to enzymes (Regand, Chowdhury, Tosh, Wolever, \& Wood, 2011). Due to its gelforming characteristics, it improves glucose tolerance through increasing volume of the alimentary bolus. As a consequence, it slows down the gastric emptying rate as well as delays the glucose absorption rate into blood by forming an intermediate film between the brush border enzymes and intestinal lumen (Schuchardt et al., 2016).

In addition, b-glucan which is a water-soluble polymer has a high water holding capacity. It absorbs water more easily than the starch granules and reduces the ability of starch to gelatinise during cooking. As a result, there is a reduced occurrence of starch molecular order disorder and a reduced surface area for the breakdown of starch into glucose, which linked to the reduced postprandial blood glucose level peak (Regand et al., 2011). In this study, the levels of b-glucan significantly $(P<0.05)$ increased with increased levels of PSC powder, which is believed to have resulted in the lowering of the $\mathrm{GI}$. These findings were in agreement with the study of Radovanovic et al. (2015), who reported that the DF as well as inulin (SDF) content from dry Jerusalem artichoke had a role in lowering the $\mathrm{Gl}$ of food.

Protein as one of the macronutrients in the food can also affect the glycaemic response. It is exceptionally efficacious in promoting release of gut peptides, which are biologically occurring short chains of amino acid monomers linked by peptide bonds. Release of insulin which act as an anabolic hormone is stimulated to allow for a greater uptake of amino acids into muscle tissue, thereby subsequently regulates blood glucose level (Dimitriadis, Mitrou, Lambadiari, Maratou, \& Raptis, 2011). In this study, the addition of PSC powder increased the level of proteins, indicating that the level of protein was one of the factors for the lowering of GI. Similarly, Giuberti et al. (2015) documented that the Gl decreased as new bean flour increased in pasta formulations.

Considering the fact that impaired glucose tolerance after meals is a key feature of diabetes, the inclusion of this low-GI biscuit in daily diet benefits diabetic individuals by lowering their postprandial blood glucose level peak. Furthermore, consumption of low-GI biscuit improves glucose tolerance, reduces insulin demand and controls body weight, which responsible for the reduced risk of developing type 2 diabetes. Therefore, it is appropriate to be consumed by general populations. Previous study indicated that intake of low-GI biscuits able to reduce the glycaemic response to a similar extent in both healthy individuals and individuals with diabetes (Jenkins et al., 2008). The low-Gl biscuit should be component of standard diet, which is consumed in normal quantities and on regular basis by all consumers.

\subsection{Sensory acceptability}

Enrichment of bakery products with DF can cause noticeable losses in aroma, texture, appearance, flavour and mouthfeel. Considering a novel food appropriately formulated from a nutritional and healthy perspective should also be satisfactory and pleasant at consumption, evaluating the sensory acceptability of the PSCformulated biscuit is necessary. Sensory scores of all attributes for biscuits added with PSC powder are illustrated in Fig. 3.

Among all PSC-based biscuits, biscuits containing 4\% PSC powder recorded the highest scores for aroma (5.38 \pm 1.17$)$, colour $(5.03 \pm 1.25)$, flavour $(5.28 \pm 1.18)$ and overall acceptance $(5.37 \pm 1.28)$. It could be believed that the $4 \%$ PSC biscuit had a milder PSC flavour, lighter colour and softer texture, similar to that of the control biscuit. However, in the case of the $12 \%$ PSC powder addition, consumers assigned the lowest score $(P<0.05)$ possibly attributed to the higher degree of firmness and the stronger PSC aroma and flavour as well as the darker surface colour of the biscuits. It could be stated that a small amount of PSC powder added can aid in bringing a more desirable aroma, colour and flavour when compared with the control biscuit. Nevertheless, with higher amounts of PSC powder added, undesirable results were attained. It is attributed to the original brownish-yellow colour and

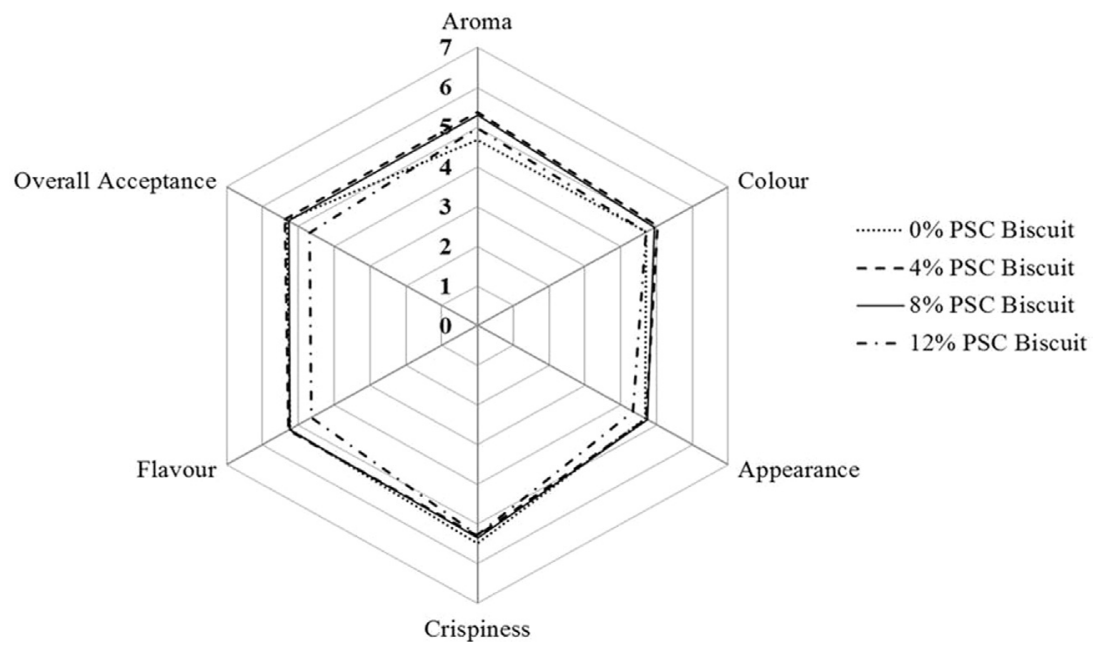

Fig. 3. Sensory acceptability of biscuits formulated with PSC powder $(n=60)$. 
distinguished flavour of the PSC powder. This is in agreement with a study conducted using oat, wheat and barley bran in biscuit formulations (Sudha, Vetrimani, \& Leelavathi, 2007). Results showed that the crumb colour darkened with an increase in the level of bran.

In terms of appearance, crispiness, flavour and overall acceptance, PSC powder could be incorporated into a biscuit up to $8 \%$, as there were no significant differences compared to the control and $4 \%$ PSC biscuit. In addition, consumers were unable to differentiate the crispiness (ranged from $5.26 \pm 1.36$ to $5.48 \pm 1.07$ ) of biscuits to which different levels of PSC powder were added, as there was no marked difference among all biscuit formulations. A similar effect was observed when different levels $(0,10,20$ and $30 \%)$ of pea flour were supplemented in cracker biscuits (Kohajdová, Karovičová, \& Magala, 2013), in which fewer consumers reported satisfaction with higher levels of DF added into bakery products. Since functional food with acceptable sensory characteristics is of major concern for fulfilling consumers' expectations, the addition of $8 \%$ PSC powder is the most effective way to produce palatable and low-GI biscuits without negatively changing its desirable sensory characteristics.

\section{Conclusion}

In summary, this study demonstrated that the use of an $8 \%$ PSC powder is an innovative approach to developing a palatable highDF and low-GI biscuit. The low GI of this biscuit could be attributed to the high DF level, which, in turn, reduced starch pasting viscosities, reduced starch gelatinisation enthalpy value, disturbed starch granules' morphology and reduced starch digestibility after the incorporation of PSC powder into biscuits. For future work, a study on the long-term intervention of this low-GI food is recommended to be done through large-scale population trials, particularly the diabetic population. PSC powder, which represents as a novel alternative DF-rich and low-Gl food ingredient, could be widely utilised in developing various PSC-based functional foods.

\section{Authors' contributions}

SHN carried out research experiment, data analysis and wrote the manuscript. SDR and WANWA participated in the design and performance of this study as well as edited the manuscript. WRWI defined the intellectual content, edited the manuscript and guarantor for the entire experiment. All authors read and approved the final manuscript.

\section{Conflict of interest}

The authors declare that no conflict of interest existed.

\section{Acknowledgements}

The work was supported by Innovation Seed Fund of Universiti Sains Malaysia [Grant no: 1001/PPSK/AUPI00222]. We are also thankful to the staff members from nutrition lab and food preparation lab in Universiti Sains Malaysia for giving laboratory facilities.

\section{References}

Adebowale, A. A., Adegoke, M. T., Sanni, S. A., Adegunwa, M. O., \& Fetuga, G. O. (2012). Functional properties and biscuit making potentials of sorghum-wheat flour composite. American Journal of Food Technology, 7(6), 372-379.

Aminah, A., \& Tan, C. C. (2001). Optimization of reduced calorie tropical mixed fruits jam. Food Quality and Preference, 12(1), 63-68.

AOAC (1990). Total dietary fibre in foods. Enzymatic-gravimetric method. Official methods of analysis (15th ed.). Washington, DC, USA: Association Official Analytical Chemists
AOAC (1995). Glucose, fructose, sucrose, and maltose in pre-sweetened cereal. Official methods of analysis (16th ed.). Washington, DC, USA: Association Official Analytical Chemists.

AOAC (1996). Official methods of analysis (16th ed.). Washington, DC, USA Association Official Analytical Chemists.

Ayo, J. A., Ayo, V. A., Nkama, I., \& Adewori, R. (2007). Physiochemical, in-vitro digestibility and organoleptic evaluation of "acha" wheat biscuit supplemented with soybean flour. Nigerian Food Journal, 25(1), 77-89.

BeMiller, J. N. (2011). Pasting, paste, and gel properties of starch-Hydrocolloid combinations. Carbohydrate Polymers, 86(2), 386-423.

Brennan, C. S., \& Tudorica, C. M. (2008). Evaluation of potential mechanisms by which dietary fibre additions reduce the predicted glycaemic index of fresh pastas. International Journal of Food Science \& Technology, 43(12), 2151-2162

Chen, J., \& Raymond, K. (2008). Beta-glucans in the treatment of diabetes and associated cardiovascular risks. Vascular Health and Risk Management, 4(6), 1265-1272.

Cheung, P. C. K. (2013). Mini-review on edible mushrooms as source of dietary fiber: preparation and health benefits. Food Science and Human Wellness, 2(3), 162-166.

Collar, C., Santos, E., \& Rosell, C. M. (2006). Significance of dietary fiber on the viscometric pattern of pasted and gelled flour-fiber blends. Cereal Chemistry, 83(4), 370-376.

Dimitriadis, G., Mitrou, P., Lambadiari, V., Maratou, E., \& Raptis, S. A. (2011). Insulin effects in muscle and adipose tissue. Diabetes Research and Clinical Practice, 93 $52-59$.

Englyst, K. N., Vinoy, S., Englyst, H. N., \& Lang, V. (2003). Glycaemic index of cereal products explained by their content of rapidly and slowly available glucose. British Journal of Nutrition, 89(3), 329-340.

FAOMHO (1998). Carbohydrates in human nutrition. Report of a Joint FAO/WHO Expert Consultation. FAO Food and Nutrition Paper, 66, 1-140.

Foschia, M., Peressini, D., Sensidoni, A., \& Brennan, C. S. (2013). The effects of dietary fibre addition on the quality of common cereal products. Journal of Cereal Science, 58(2), 216-227.

Giuberti, G., Gallo, A., Cerioli, C., Fortunati, P., \& Masoero, F. (2015). Cooking quality and starch digestibility of gluten free pasta using new bean flour. Food Chemistry, 175, 43-49.

Guariguata, L., Whiting, D. R., Hambleton, I., Beagley, J., Linnenkamp, U., \& Shaw, J. E. (2014). Global estimates of diabetes prevalence for 2013 and projections for 2035. Diabetes Research and Clinical Practice, 103(2), 137-149.

Imran, M. M., Raja, M. M., Basith, M. A., \& Asarudin, A. (2011). Determination of total phenol, flavonoid and antioxidant activity of edible mushrooms Pleurotus florida and Pleurotus eous. International Food Research Journal, 18, 574-577.

Jenkins, A. L., Jenkins, D. J. A., Wolever, T. M. S., Rogovik, A. L., Jovanovski, E., Bozikov, V., et al. (2008). Comparable postprandial glucose reductions with viscous fiber blend enriched biscuits in healthy subjects and patients with diabetes mellitus: Acute randomized controlled clinical trial. Croatian Medical Journal, 49(6), 772-782.

Kohajdová, Z., Karovičová, J., \& Magala, M. (2013). Rheological and qualitative characteristics of pea flour incorporated cracker biscuits. Croatian Journal of Food Science and Technology, 5(1), 11-17.

Lattimer, J. M., \& Haub, M. D. (2010). Effects of dietary fiber and its components on metabolic health. Nutrients, 2(12), 1266-1289.

Mamat, H., Abu Hardan, M. O., \& Hill, S. E. (2010). Physicochemical properties of commercial semi-sweet biscuit. Food Chemistry, 121(4), 1029-1038.

McCleary, B. V., \& Glennie-Holmes, M. (1985). Enzymic quantification of (1-3)(14)-b- D-glucan in barley and malt. Journal of the Institute of Brewing, 91, 285-295.

Mira, I., Eliasson, A. C., \& Persson, K. (2005). Effect of surfactant structure on the pasting properties of wheat flour and starch suspensions. Cereal Chemistry, 82 (1), 44-52.

Ng, S. H., Mohd Zain, M. S., Zakaria, F., Wan Ishak, W. R., \& Wan Ahmad, W. A. N. (2015). Hypoglycaemic and antidiabetic effect of Pleurotus sajor-caju aqueous extract in normal and streptozotocin-induced diabetic rats. BioMed Research International. http://dx.doi.org/10.1155/2015/214918.

Ng, S. H., \& Wan Rosli, W. I. (2014). Effect of cinnamon powder addition on nutritional composition, physical properties and sensory acceptability of butter biscuits. Malaysian Journal of Nutrition, 20(2), 24-253.

Norimah, A., Safiah, M., Jamal, K., Haslinda, S., Zuhaida, H., Rohida, S., et al. (2008). Food consumption patterns: $\quad$ Findings from the Malaysian Adult Nutrition Survey (MANS). Malaysian Journal of Nutrition, 14(1), 25-39.

Radovanovic, A., Stojceska, V., Plunkett, A., Jankovic, S., Milovanovic, D., \& Cupara, S. (2015). The use of dry Jerusalemartichoke as a functional nutrient in developing extruded food with low glycaemic index. $\quad$ Food Chemistry, 177 81-88.

Regand, A., Chowdhury, Z., Tosh, S. M., Wolever, T. M., \& Wood, P. (2011). The molecular weight, solubility and viscosity of oat beta-glucan affect human glycemic response by modifying starch digestibility. Food Chemistry, 129(2), 297-304.

Ren, X., Chen, J., Molla, M. M., Wang, C., Diao, X., \& Shen, Q. (2016). In vitro starch digestibility and in vivo glycemic response of foxtail millet and its products Food \& Function, 7(1), 372-379.

Schuchardt, J. P., Wonik, J., Bindrich, U., Heinemann, M., Kohrs, H., Schneider, I., et al. (2016). Glycemic index and microstructure analysis of a newly developed fiber enriched cookie. Food \& Function, 7(1), 464-474.

Shah, S., Ghosh, D., Mallick, S. K., Sarangi, I., Bhutia, S. K., Banerje, I., et al. (2007) Immunomodulatory and antitumor activities of water-soluble proteoglycan 
isolated from the fruiting bodies

of culinary-medicinal oyster mushroom

Pleurotus ostreatus. International Journal of Medicinal Mushroom, 9, 123-138.

Simsek, S., \& Nehir El, S. (2015). In vitro starch digestibility, estimated glycemic index and antioxidant potential of taro (Colocasia esculenta L. Schott) corm. Food Chemistry, 168, 257-261.

Singh, J., Dartois, A., \& Kaur, L. (2010). Starch digestibility in food matrix: A review. Trends in Food Science \& Technology, 21(4), 168-180.

Sozer, N., Cicerelli, L., Heiniö, R. L., \& Poutanen, K. (2014). Effect of wheat bran addition on in vitro starch digestibility, physico-mechanical and sensory properties of biscuits. Journal of Cereal Science, 60(1), 105-113.

Sudha, M. L., Vetrimani, R., \& Leelavathi, K. (2007). Influence of fibre from different cereals on the rheological characteristics of wheat flour dough and on biscuit quality. Food Chemistry, 100(4), 1365-1370.
Tudorica, C. N., Kuri, V., \& Brennan, C. S. (2002). Nutritional and physicochemical characteristics of dietary fiber enriched pasta. Journal of Agriculture and Food Chemistry, 50(2), 347-356.

Vujic', L., Vitali Čepo, D., \& Vedrina Dragojevic ', I. (2015). Impact of dietetic tea biscuit formulation on starch digestibility and selected nutritional and sensory characteristics. LWT - Food Science and Technology, 62, 647-653.

Wan Rosli, W. I., \& Aishah, M. S. (2012). Pleurotus sajor-caju (PSC) improves nutrient contents and maintains sensory properties of carbohydrate-based products. International Journal of Biological, Biomolecular, Agricultural, Food and Biotechnological Engineering, 6(3), 87-89.

Wu, N. J., Chiou, F. J., Weng, Y. M., Yu, Z. R., \& Wang, B. J. (2014). In vitro hypoglycemic effects of hot water extract from Auricularia polytricha (wood ear mushroom). International Journal of Food Sciences and Nutrition, 65(4), 502-506. 